\title{
Zytokintherapie bei malignen Hauterkrankungen und insbesondere beim malignen Melanom
}

\author{
M. Beyeler \\ R. Dummer
}

\author{
Cytokine Therapy in the Treatment of Malignant Skin Diseases - Especially \\ Concerning the Malignant Melanoma
}

\section{Zusammenfassung}

Zytokine sind hormonähnliche Glykoproteine mit para- oder autokriner Wirkung. Zytokine regulieren Immunreaktionen und Entzündungsvorgänge. Ihren Effekt üben Zytokine durch Bindung an spezifische Zellrezeptoren aus, welche eine Signalübertragung in die Zelle und schließlich den biologischen Effekt bewirken. Die Zytokine können unterteilt werden in Interleukine, Interferone, colony-stimulating Factors, Tumornekrosefaktoren und transforming growth Factor $\beta$, wobei in der Dermatologie vor allem Interferon- $\alpha,-\beta,-\gamma$, Interleukin-2, GM-CSF sowie Interleukin-12 zur Anwendung kommen. Nebenwirkungen einer Zytokintherapie bestehen meist aus grippalen Beschwerden, außerdem kann es zu Leukopenien sowie Leberwerterhöhungen kommen. Die Nebenwirkungen lassen sich durch Paracetamol und Dosisanpassung meist gut angehen. Gewisse Zytokine wie z.B. Interferon- $\alpha$ sind für bestimmte dermatologische Erkrankungen bereits registriert, während andere noch in klinischen Studien erprobt werden. Dieser Artikel beschränkt sich auf die Anwendung bei malignen Hauterkrankungen, wo verbesserte Therapieoptionen dringend nötig wären.

\section{Abstract}

Cytokines are glycoproteins similar to hormones with a paracrine or autocrine effect. Cytokines regulate immune reactions and inflammatory processes. They bind to specific cell receptors which then activate a signalling cascade and in the end a biological effect. Cytokines can be divided into interleukins, interferons, colony-stimulating factors, tumor necrosis factors and transforming growth factor $\beta$. In dermatology, above all interferon- $\alpha,-\beta$, $-\gamma$, interleukin-2, GM-CSF and interleukin-12 are used. Side effects of a cytokine therapy include flu-like symptoms, leukopenia and increase of liver enzymes. In most cases these side effects can easily be treated with paracetamol and dose reduction. Some cytokines such as interferon- $\alpha$ are already registered whereas others are applied in clinical trials. This article is limited to cytokine therapy of malignant skin tumors, where better treatment results are needed.

\section{Einleitung}

Auf der Suche nach besseren Behandlungsmöglichkeiten für das Melanom und andere Hautneoplasien ergaben sich neue Therapieansätze mit dem Ziel, die körpereigene Immunabwehr gegen die Tumorzellen zu richten. Bereits in den 80er-Jahren starteten erste Versuche mit Interferon- $\alpha$ (INF- $\alpha$ ) zur Behandlung dieses bösartigen Tumors, welche im Verlauf der Jahre durch Studien mit weiteren Zytokinen (Interleukine, hämatopoetische Growth
Factors) ergänzt wurden. Andere Versuche zeigten außerdem, dass sich diese Zytokine auch für die Behandlung weiterer Hauttumoren oder auch entzündlichen Hautläsionen eigneten.

Dieser Artikel soll einen Überblick über die Anwendung der Zytokine in der Dermatoonkologie vermitteln. Unzählige Studien zu diesem Gebiet sind aktuell noch unvollendet und werden unser Wissen zu diesem spannenden Thema noch erweitern. 
Zytokine - ein Oberbegriff für Interleukin $1-23$, Interferon $\alpha, \beta$ und $\gamma$, colony-stimulating Factors (M-CSF, G-CSF, GM-CSF, Erythropoetin), Tumornekrosefaktor $\alpha$ und $\beta$ sowie transforming growth Factor $\beta$ - sind biologisch aktive, hormonähnliche Glykoproteine, die von bestimmten Zellen gebildet werden und bei entsprechenden, rezeptortragenden Zielzellen einen biologischen Effekt ausüben. Aufgrund des Zelltyps, die präferenziell aber keineswegs exklusiv - ein bestimmtes Zytokin freisetzt, werden Unterbegriffe verwendet. Monokine z.B. sind vor allem von Makrophagen/Monozyten gebildete Zytokine. Lymphokine sind Zytokine, die - oft neben vielen anderen Effekten - entweder von Lymphozyten gebildet werden und/oder auf Lymphozyten wirken, ein bereits eingeschränkter Begriff. Mit Interleukinen werden Zytokine bezeichnet, die u.a. wesentlich die spezifische Immunantwort beeinflussen. Sie sind von einem internationalen Komitee nummeriert worden. Den Zytokinen sind bestimmte Eigenschaften gemeinsam [1]:

- zellregulatorische Eiweiße

- MG unter 60000 Dalton (meist unter 25000)

- lokal produziert, mit kurzer Halbwertszeit (Minuten)

- wirken in pikomolarer Konzentration

- wirken meist parakrin (in Nähe des Produktionsortes) und oft auch autokrin (auf die produzierende Zelle)

- interagieren mit hochaffinen Zellrezeptoren

- induzieren auf gleichen Targetzellen ähnliche Effekte (z.B. Mediatorfreisetzung, Regulation der Expression von Adhäsionsmolekülen)

In den letzten Jahren sind viele Zytokine voll sequenziert und gentechnologisch hergestellt worden. Die Messenger-RNA's sind bekannt und von vielen Zytokinen auch die entsprechenden Rezeptoren.

\section{Biologische Wirkung von Zytokinen}

Zytokine bilden ein „Netzwerk“, welches sowohl die unspezifischen als auch spezifischen Immunreaktionen sowie die Entzündungsvorgänge als Ganzes reguliert, d.h. für deren örtliche und zeitliche Ausdehnung entscheidend ist. Ebenfalls sind sie für bestimmte systemische Entzündungszeichen wie Fieber, Abgeschlagenheit und wahrscheinlich auch für gewisse Schockzustände usw. verantwortlich. Im Weiteren beeinflussen Zytokine auch das neuroendokrine System. Die genauen Zusammenhänge sind aber erst teilweise bekannt.

a) Spezifische Immunität: Zytokine sind wichtige Modulatoren der Immunantwort im engeren Sinn, d.h. sie sind mitentscheidend über Art und Ausmaß (örtlich und zeitlich) einer spezifischen humoralen oder zellulären Immunantwort, indem sie APC, T-Helfer- und zytotoxische T-Lymphozyten sowie B-Zellen aktivieren und/oder differenzieren und/oder expandieren. Zytokine sind also wesentliche Faktoren der T-T-Hilfe und der T-B-Hilfe.

b) Unspezifische Immunantwort: Einige Lymphokine nehmen insofern eine spezielle Position ein, als dass sie im Rahmen einer Ag-abhängigen oder polyklonal ausgelösten Immunreaktion auf Stufe APC und T-Helfer-Lymphozyt gebildet werden, aber dann Ag-unabhängig die unspezifische Immunantwort wesentlich beeinflussen. So aktiviert Interferon- $\gamma$ die Natural Killerzellen (NK) zu LAK-Zellen (lymphokine-activated killer cells) und macht aus Makrophagen/Monozyten Killerzellen. NK-/LAK-Zellen, Killerzellen und wahrscheinlich auch $\gamma / \delta$-Zellen sind wichtige Elemente der frühen Immunabwehr, d. h. sie wirken, bevor die spezifische Immunreaktion zum Tragen kommt.

c) Entzündung und Reparation: Zytokine spielen zusätzlich eine wesentliche Rolle als generelle Entzündungs- und auch Reparationsmediatoren, indem sie viele andere Zellen beeinflussen, so z.B. Eosinophile, Mastzellen, neutrophile Granulozyten, Endothelzellen, Epithelzellen, Fibroblasten, Astrozyten, Osteoklasten, Osteoblasten, Hepatozyten u.a.m. Die Wirkung reicht von Chemotaxis zur Aktivation, Differenzierung und zur Proliferation dieser Zellen, die ihrerseits über ihre eigenen Mediatoren die Entzündung resp. Reparation beeinflussen.

\section{Wirkungsweise}

a) Zytokine wirken nur auf Zellen, die die entsprechenden Rezeptoren haben. Oft führt ein Zytokin zur verstärkten Expression von Rezeptoren für andere Zytokine. So kann es zu kaskadenähnlichen Wirkungen kommen. Die meisten Zytokine verlassen die produzierende Zelle in aktiver Form. Ausnahme ist TGF- $\beta$, welches als großmolekulare Vorstufe freigesetzt wird und dann einer zusätzlichen Aktivation bedarf. Der genaue Mechanismus ist noch unklar (pH-Veränderung, Proteasen).

b) Unter physiologischen Umständen wirken Zytokine lokal wie sich physiologischerweise auch die meisten Immunreaktionen lokal abspielen. Bei überschießender oder generalisierter Reaktion können sie aber auch zu schweren „Nebenerscheinungen“, ja sogar zum Organversagen resp. Tod führen. Dies gilt z. B. für IL-1 und TNF im septischen Schock: Produkte von Mikroorganismen binden an die so genannten „toll-like Receptors“, transmembranöse Signalrezeptoren, welche anschließend die Produktion von Zytokinen aktivieren und so einerseits zur Abwehr des Mikroorganismus beitragen, andererseits aber auch zu Gewebeschaden inklusive septischem Schock mit Zelluntergang führen können [2 -4].

Um therapeutisch Effekte/Konzentrationen eines gegebenen Lymphokins lokal am Ort des Geschehens zu erreichen, müssen systemisch so hohe Dosen gegeben werden, dass die $\mathrm{Ne}$ benerscheinungen in den Vordergrund treten.

c) Viele Lymphokine wirken synergistisch oder z.B. gestaffelt auf die B-Lymphozyten zur Plasmazellentwicklung (IL-1 bis 7), einige aber regulativ im Sinne der Inhibition. Z.B. IFN- $\gamma$ und IL-4 aktivieren beide synergistisch T-Lymphozyten, zeigen aber verschiedene Wirkung auf die Isotypenselektion von B-Lymphozyten. So inhibiert IFN- $\gamma$ den stimulierenden Effekt von IL-4 auf die Bildung von IgE.

Zytokine üben ihren Effekt durch Bindung an Oberflächenrezeptoren aus, welche mit der JAK-Familie (Janus-Kinase), zytoplasmatischen Tyrosinkinasen, assoziiert sind. Zytokininduzierte Rezeptordimerbildung führt zur Aktivierung von JAK, es kommt zu Tyrosinphosphorylierungen der zytoplasmatischen Rezeptordomänen und zur Aktivation verschiedener Signalproteine inklusive Mitglieder der STAT-Familie (signal transducer and activator of transcription) [5]. Aktivierte STAT formen Dimere, migrieren 
in den Zellkern, binden an spezifische Stellen von Promotoren und aktivieren die Transkription dieser Gene. Es gibt stimulierende und inhibierende Regulierungen [6].

Wichtigste Zytokine für die Anwendung in der Dermatologie sind Interferone ( $\alpha-, \beta-, \gamma$-Interferon), Interleukin-2, GM-CSF und Interleukin-12.

\section{Interferone}

\section{Wirkmechanismen}

IFN- $\alpha$ und $-\beta$ werden vor allem von Makrophagen/Monozyten gebildet (= Monokine). Sie wirken antiviral und antitumoral, sie erhöhen die Expression von MHC-I-Antigenen, sind teilweise antagonistisch zu IFN- $\gamma$, inhibieren LAK-Zellen und stimulieren NKZellen. Anders formuliert: IFN- $\alpha$ ist beteiligt an Regulation der Zelldifferenzierung, Inhibition der Tumorzellproliferation, proinflammatorischer Immunmodulation sowie Antiangiogenese [7].
IFN- $\gamma$ wird vor allem von T-Helfer-Lymphozyten gebildet (= Lymphokin). IFN- $\gamma$ wirkt antiviral und antiproliferativ, es erhöht die Expression von MHC-I-Antigenen und - wichtig - auch von MHC-II-Antigenen auf APC, Endothelzellen u.a.m. (verbesserte Ag-Präsentation), IFN- $\gamma$ erhöht die NK-Aktivität und „,killing“-Kapazität von Makrophagen/Monozyten und antagonisiert die Wirkung von IL-4 bezüglich IgE-Produktion.

Signalübertragung in die Zelle (Abb. 1 u. 2)

Es gibt zwei Sorten von Interferon-Rezeptoren: einer für alle Interferon- $\alpha$-Subtypen und Interferon- $\beta$ sowie ein Rezeptor für Interferon- $\gamma[8]$ (Abb. 1). IFN- $\alpha$ bindet an IFN- $\alpha-$ R1 und -2 und aktiviert dadurch JAK1 und Tyk 2, welche anschließend über STAT1 bis $3 \mathrm{im}$ Zellkern auf die DNA wirken. IFN- $\gamma$ bindet an einen Zellrezeptor IFN- $\gamma$-R1, an welchen sich anschließend IFN- $\gamma$-R2 anlagert. Dadurch werden zwei Moleküle JAK1 und zwei Moleküle JAK2 aktiviert und wirken via STAT1 auf die DNA [9-11] (Abb. 2). JAK1 wird somit durch IFN- $\alpha$ und $-\gamma$ aktiviert, JAK2 nur durch IFN- $\gamma[12]$.

\section{Indikationen}

In der adjuvanten Behandlung des malignen Melanoms führt IFN- $\alpha$ in s.c.-Applikation zu einer Verlängerung des rezidivfreien Intervalls $[13,14]$. Dies gilt sowohl für Studien mit niedriger Dosis wie auch Hochdosis-IFN- $\alpha$-Applikation.

Da Hochdosis-IFN- $\alpha$-Therapie in der Behandlung der kutanen T-Zell-Lymphome eine hohe Toxizität aufweist und niedrige Dosen eine unzureichende Remissionsdauer erzielen, untersuchte man Kombinationstherapien. Kombinierte PUVA-IFN- $\alpha$-Behandlung wird zur Therapie der kutanen T-Zell-Lymphome in den Stadien IA-IIB eingesetzt [15]. Auch Kombinationen mit Retinoiden kommen zum Einsatz.

Zur Behandlung von B-Zell-Lymphomen stellt IFN- $\alpha$ eine Alternative zur Exzision oder Radiatio dar.

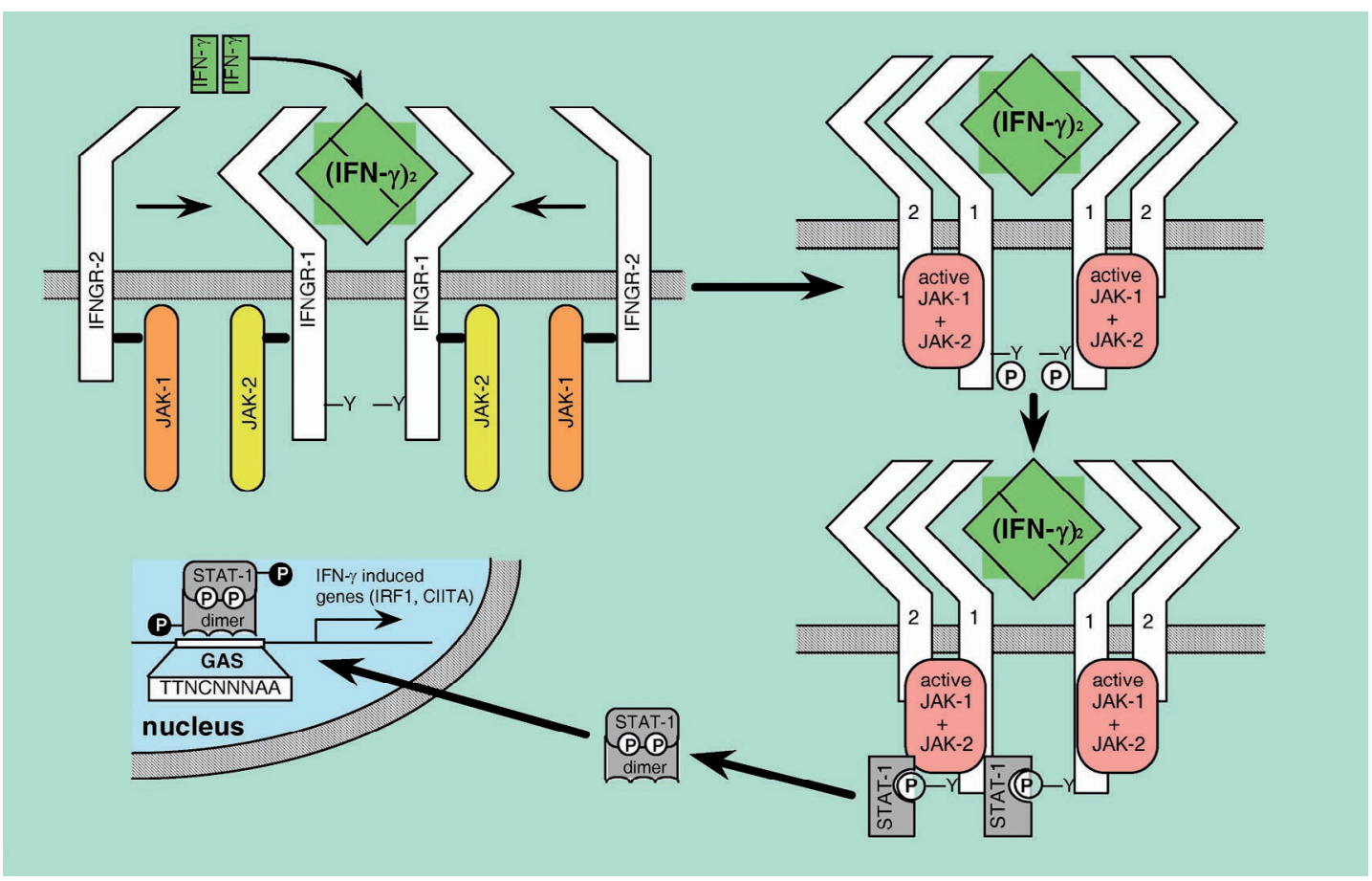

Abb. 2 Signalübertragung von Interferon- $\gamma$. 
Bei inoperablem Status bei Spinaliomen und Basaliomen ist die lokale IFN- $\alpha$-Therapie eine mögliche Option [16]. Lippmann erzielte nach Einsatz von IFN- $\alpha$ in Kombination mit 13-cis-Retinsäure 25\% komplette Remissionen bei inoperablen Spinaliomen [17]. Chimenti konnte bei Basaliomen durch intra- und periläsionäre Injektion von IFN- $\alpha$ in $67 \%$ eine komplette Remission erzielen [18].

Auch beim Merkelzell-Karzinom kann bei Rezidiv oder Progress der lokale Einsatz von IFN- $\alpha$ erwogen werden. Der Einsatz von Zytokinen bei Merkelzell-Karzinom hat hauptsächlich bei inoperablem Befund eine Bedeutung.

In einem Fall eines Angiosarkoms wurde eine erfolgreiche Therapie mit INF- $\alpha .2 b$ in Kombination mit Interleukin-2 durchgeführt [19].

INF- $\beta$ hat die stärkste antiproliferative Aktivität gegen Melanomzelllinien. In einer Studie wurde eine Transfektion von Melanomzellen mit kationischen Liposomen, welche das Gen für INF- $\beta$ enthielten, durchgeführt und so eine Hemmung des Zellwachstums der Melanomzellen erreicht [20]. Auch in anderen Studien wurde INF- $\beta$ erfolgreich gegen Melanom eingesetzt [21,22].

Zwei Patientinnen mit bowenoider aktinischer Keratose wurden mit intraläsionärer Injektion von Interferon- $\beta$ behandelt und erreichten eine komplette Remission [23].

INF- $\gamma$ (und auch IFN- $\alpha$ oder IFN- $\beta$ ) wurde erfolgreich eingesetzt in der Behandlung von Genitalwarzen, Psori-Arthritis, Psoriasis vulgaris, malignem Melanom, bowenoider Papulose, M. Behçet, Basaliom, Herpes simplex rezidivans, Epidermodysplasia verruciformis sowie Mycosis fungoides [24-27].

Bisherige Therapiestudien konnten weder Behandlungsvorteil noch eine verbesserte Überlebensrate für IFN- $\gamma$ in der Therapie des malignen Melanoms nachweisen. Es wird vor allem in Kombination mit TNF- $\alpha$ und Mephalan im Rahmen von Extremitätenperfusionen eingesetzt [28].

\section{Pharmakologische Veränderungen (pegylierte Interferone)}

Als Nebenwirkungen einer Interferontherapie ergeben sich grippeähnliche Symptome wie Fieber, Schüttelfrost, Kopfschmerzen, Müdigkeit, Myalgien und Übelkeit, außerdem Leukozytopenie und Thrombozytopenie. Durch molekulare Veränderungen der Interferone versuchte man, ein besseres Wirkungsprofil zu erreichen. Durch Polyethylglycolisierung des Interferons ergibt sich ein erhöhtes Molekulargewicht mit verlängerter Halbwertszeit ( $54 \mathrm{~h}$ vs. $8,1 \mathrm{~h}$ ), verminderter Antigenizität, reduzierter Anfälligkeit auf Proteolyse sowie einer verbesserten Verträglichkeit. Es werden so stabilere Serumspiegel erreicht. PEG-Intron kann einmal wöchentlich verabreicht werden, während das herkömmliche INF- $\alpha$ dreimal wöchentlich injiziert werden muss. Das pegylierte Interferon ist zur Behandlung der chronischen Hepatitis C zugelassen und scheint auch in der dermatologischen Anwendung effizient.

Beim metastasierenden malignen Melanom kann PEG-IFN anhaltende Remissionen induzieren [29].

\section{Ausblick: gentherapeutische Ansätze}

Einerseits werden die natürlich auftretenden Interferone $\alpha, \beta, \gamma$ therapeutisch eingesetzt, andererseits werden seit Entschlüsselung der Gene auch rekombinante Interferone hergestellt. Die $\alpha$-Familie besteht dabei aus mehreren Subtypen, welche sich durch einzelne Aminosäuren unterscheiden.

Es werden Wege gesucht, eine hohe lokale Konzentration der Interferone zu erreichen ohne hohe systemische Gaben. Die Interferongene wurden in Viren transfiziert und diese dann lokal appliziert. Ein anderer gentechnischer Ansatz sind Viren, welche Melanomantigene exprimieren und so eine Abwehrantwort induzieren.

\section{Interleukin-2}

\section{Wirkmechanismen}

IL-2 ist ein Zytokin, das vor allem von T-Helfer-Lymphozyten gebildet wird [30]. Es zeigt eine autokrine Wirkung auf aktivierte T-4-Lymphozyten, ist ein Wachstumsfaktor für zytotoxische T-Lymphozyten und LAK-Zellen, erhöht die NK-Aktivität und „killing“-Kapazität von Makrophagen/Monozyten und beeinflusst die B-Lymphozyten. Es induziert die Bildung weiterer Zytokine (TNF- $\alpha$, IFN- $\gamma$, IL-5, IL-6, GM-CSF, M-CSF). Interleukin-2 wirkt also nicht direkt antitumoral, sondern entfaltet seine Wirkung durch die Stimulierung der zytotoxischen Mechanismen der T-Lymphozyten und NK-Zellen.

\section{Signalübertragung in die Zelle}

Der IL-2-Rezeptor besteht aus 3 Ketten $(\alpha, \beta, \gamma)$, welche IL- 2 binden [31]. Die $\beta$ - und $\gamma$-Ketten sind für die Signaltransduktion essenziell. Sie bestehen aus einem extrazellulären, transmembranösen und einem zytoplasmatischen Anteil, welche homolog sind zu Rezeptoren von hämatopoetischen Growth Factors.

Interleukin-2-Bindung führt zu einer Heterodimerbildung der $\beta$ und $\gamma$-Kette und anschließend zur Aktivierung von Proteintyrosinkinase (PTK) und weiteren Signalmolekülen wie glycolisiertem Phosphatidylinositol (GPI), Phosphatidylinositol-3-Kinase (PI3K), p74c-raf und p21ras [32].

Die $\alpha$-Kette ist verantwortlich für die Spezies-Spezifität der Bindung von Interleukin-2 [33].

Der zytoplasmatische Teil der $\beta$-Kette besteht aus mindestens 2 verschiedenen Regionen: einer Serin-reichen Region, verantwortlich für Mitosesignale, und einer „sauren“ Region, welche die p561ck-PTK aktiviert. So führt IL-2-Stimulation einerseits zu Tyrosinphosphorylierungen (u.a. von STAT3 und STAT5), welche nachher die Induktion von c-fos, c-jun und anderen Genen herbeiführen, andererseits kommt es zur Induktion c-myc-Genen (Protoonkogenen) [34,35].

Die $\gamma$-Kette findet sich auch in Rezeptoren für IL-7 und IL-4 wieder [36] sowie IL-9 und IL-15. Die $\gamma$-Kette ist mit der JAK3-Tyrosinkinase verbunden. JAK3 aktiviert JAK1 und zusammen mit anderen Tyrosinkinasen phosphorylisieren sie den Rezeptor, welcher anschließend andere Signalmoleküle binden kann wie z.B. PI3-Kinase [37,38]. 


\section{Indikationen}

Interleukin-2 wird vor allem in Kombination mit INF- $\alpha$ angewendet. Es wird hauptsächlich zur Therapie des fortgeschrittenen malignen Melanoms (Stadium IV) benutzt, zum Teil in Kombination mit Chemotherapeutika. Über die beste Applikationsart sind sich die Forscher noch nicht einig. Zur Diskussion stehen s.c., i.v., verzögerte Freisetzung als 1l-2-Liposomen, lokal intratumoral oder auch inhalativ bei Lungenmetastasen.

Einen zweiten Anwendungsschwerpunkt stellen die Vakzinierungen dar. Es wird versucht, eine Hypersensitivitätsreaktion gegen Melanomzellen zu erreichen.

Interleukin-2 kommt auch in der Behandlung kutaner T-ZellLymphome wie auch B-Zell-Lymphome (z.B. in Kombination mit Antikörpern) zum Einsatz.

Periläsionäre Injektionen von PEG-IL-2 bei Patienten mit Basaliomen ergaben gute Ansprechraten ohne signifikante Nebenwirkungen in einer Studie von Kaplan [39].

\section{Ausblick: gentherapeutische Ansätze}

Das Gen für Interleukin-2 wurde in verschiedene Viren (canary pox virus, Adenoviren) und auch Vero-Zellen transfektiert, um anschließend in den Melanommetastasen eine hohe lokale Interleukin-2-Produktion zu erreichen [40]. Belli immunisierte Patienten mit metastasierendem Melanom mit HLA-A2-kompatiblen, Interleukin-2-sezernierenden Melanomzelllinien mit dem Ziel, eine systemische Abwehrreaktion zu provozieren, welche auch ferne Metastasen betreffen würde [41].

Des Weiteren wurde ein fusioniertes Zytokin-Toxin-Molekül DAB389-IL-2 entwickelt und in der Behandlung kutaner T-ZellLymphome eingesetzt. Das Toxin hat die Aufgabe, das Zytokin möglichst nahe an die T-Zellen zu bringen und anschließend eine Immunantwort $\mathrm{zu}$ induzieren.

\section{GM-CSF}

\section{Wirkmechanismen}

Der Granulocyte-macrophage colony-stimulating factor wird von aktivierten T-Lymphozyten gebildet. Er stellt einen Wachstumsfaktor für Vorläufer von myeloiden Zellen (Granulozyten und Makrophagen/Monozyten sowie dendritischen Zellen) dar und stimuliert die Aktivität von reifen Neutrophilen, Eosinophilen, dendritischen Zellen und Makrophagen/Monozyten (u.a. Tumorantigenpräsentation, T-Zell-vermittelte Immunität). Unter dem Einfluss von GM-CSF werden Makrophagen zytotoxisch gegen menschliche Melanomzellen [42].

\section{Signalübertragung in die Zelle}

Der GM-CSF-Rezeptor ist ein Heterodimer bestehend aus 2 Untereinheiten, $\alpha$ und $\beta$ [43]. Der $\beta$-Anteil spielt auch in der Signaltransduktion von IL-5 und IL-3 eine Rolle und heißt daher $\beta$ common chain, $\beta(c)$ [44]. Der $\alpha$-Teil ist zytokin-spezifisch [45], er bindet das Zytokin, während der $\beta$-Teil für die Signaltransduktion zuständig ist [46].
Bindung von GM-CSF führt zu Dimerbildung zweier $\beta(c)$-Ketten, anschließend zu Tyrosinphosphorylation der $\beta$ (c)-Ketten. Im zytosolischen Anteil der $\beta(c)$-Ketten gibt es eine Region proximal der Zellmembran, zuständig für Mitogenese, und eine distale Region, verantwortlich für die Aktivation von ras, raf-1, mitogenactivated Proteinkinase und S6-Kinase. Die proximale Region ist zuständig für die Tyrosinphosphorylierung von JAK2, was anschließend via STAT zur Induktion der Mitogenese führt [47].

\section{Indikationen}

Mit Fokus auf die Antigenpräsentation wird GM-CSF additiv zu Vakzinen appliziert oder in Melanomzellen transfiziert. Auch periläsionäre Injektionen von rekombinantem GM-CSF bei Patienten mit Hautmetastasen bei malignem Melanom führten zu Remissionen [48].

Mahvi führte erfolgreiche Studien in der Behandlung des Sarkoms durch [49].

Bei inoperablem Merkelzell-Karzinom wurden erfolgreiche Behandlungen mit GM-CSF additiv zu Cyclophosphamid durchgeführt.

\section{Ausblick: gentherapeutische Ansätze}

Als Vektor für GM-CSF kann u.a. das canary pox virus dienen. Ziel ist eine erhöhte Tumorantigen-Präsentation durch rekrutierte dendritische Zellen und Makrophagen resultierend in verstärkter zellulärer und humoraler antitumoraler Abwehr [50].

Die Proteine der ras-Familie (Protoonkogene) sind involviert in die Signalübertragung zwischen Zelloberfläche und Nukleus. Sie regeln Zellproliferation, Differenzierung, Apoptose und Transformationen. Wenn überexprimiert oder mutiert können sie eine maligne Transformation einer Zelle induzieren. Codon 61-Mutationen von N-ras scheinen an der Melanomentstehung an sonnenexponierten Stellen involviert zu sein. In einer Studie konnten intradermale Injektionen von ras-Peptiden mit GM-CSF als Adjuvans eine zelluläre Immunantwort gegen Melanomzellen induzieren [51].

\section{Interleukin-12}

\section{Wirkmechanismen}

Interleukin-12 wird durch T-Helfer-Lymphozyten gebildet. Es ist ein wesentliches Zytokin für die Entstehung von Th-1-Lymphozyten, welche anschließend durch ihre Produktion von IL-2 und IFN- $\gamma$ die Makrophagen/Monozyten und NK-Zellen aktivieren (vor allem Abwehr intrazellulärer Mikroorganismen, d.h. zelluläre Abwehr). Interleukin-12 verstärkt so die zytolytische Aktivität und Proliferation von T- und NK-Zellen, außerdem inhibiert es die Tumorangiogenese und verbessert die Antigenerkennung. Viele Melanomzellen tragen ebenfalls IL-12-Rezeptoren. IL-12 bewirkt eine Hochregulation von HLA-Klasse-I-Molekülen und Proliferationshemmung [52].

\section{Signalïbertragung in die Zelle}

Der IL-12-Rezeptor besteht aus zwei Anteilen, $\beta 1$ und $\beta 2$, wobei beide für die Bindung von IL-12 und die Signaltransduktion zuständig sind. Die $\beta 2$-Kette enthält die intrazellulären Tyrosinan- 
teile, welche für die Aktivierung von STAT4 und auch STAT1 und 3 verantwortlich sind (via Tyrosinphosphorylierung) [53]. Die STAT-Moleküle bilden einen DNA-bindenden Komplex und induzieren im Zellkern u. a. die Bildung von IFN- $\gamma$.

\section{Indikationen}

IL-12 wird bei metastasierendem malignen Melanom im Rahmen von klinischen Studien eingesetzt. Dabei wird IL-12 in der löslichen Form sowie auch als Mikrosphären mit verzögerter Freisetzung verabreicht. Eine antitumorale Effizienzsteigerung könnte durch Kombinationen mit anderen Zytokinen wie IL-2 oder GM-CSF erreicht werden [54].

Eine zweite mögliche Indikation sind kutane T-Zell-Lymphome $[55,56]$.

\section{Ausblick: gentherapeutische Ansätze}

Um eine hohe lokale Konzentration zu erreichen, werden Plasmide (nackte DNA) eingesetzt, welche cDNA von Interleukin-12 enthalten. Diese Therapie zeigt keine signifikanten Nebenwirkungen und führt zu einem Nachweis von konstant erhöhten IFN- $\gamma$-Spiegeln im Serum.

Melanomzellen können dem Immunsystem entgehen aufgrund Fehlens von immunstimulierenden Molekülen wie B7.1 oder B7.2. IL-12 induziert antitumorale Effekte, und B7.1 und IL-12 induzieren synergistisch eine effiziente antitumorale Immunität. In einer Studie wurden Melanomzellen mittels Adenoviren mit B7.1 plus IL-12 beladen. In der Folge entwickelte sich eine starke Proliferation von mononukleären Zellen mit starker Zytokinproduktion und eine vermehrte Expression von MHC-I- und II-Antigenen sowie ICAM-I-Antigenen auf Melanomzellen [57].

\section{Imiquimod und andere synthetische Zytokininduktoren}

Neben den oben beschriebenen Zytokinen kommen zunehmend auch Zytokininduktoren zum Einsatz wie z.B. Imiquimod. Diese Substanz wirkt auf Makrophagen und dendritische Zellen und induziert die Sekretion von INF- $\alpha$, Tumornekrose-Faktor- $\alpha$ und IL-12 sowie eine Aktivation der Th1-Zellen [58,59]. Sie verbessert die Antigenpräsentation auf dendritischen Zellen und fördert auch die Synthese von Antikörpern [60]. Imiquimod wurde unter dem Handelsnamen Aldara bereits erfolgreich zur Behandlung viraler Infektionen (Human Papillomavirus von analen und genitalen Warzen, Herpes-simplex-Virus, Molluscum contagiosum), Hauttumoren (aktinische Keratose, Spinaliom, Basaliom, Lentigo maligna, Melanom, M. Bowen) und bei der Mycosis fungoides eingesetzt [61-66].

\section{Schlussfolgerung}

Angesichts der vielen Studien hat sich unser Wissen über die Wirkung und therapeutische Einsatzmöglichkeiten der Zytokine in den letzten Jahren deutlich weiterentwickelt. Dennoch sind die Resultate vor allem in der Behandlung des malignen Melanoms immer noch ungenügend, zum Teil auch widersprüchlich. Es scheint auch so, dass nur wenige Patienten von einer Immuntherapie profitieren. Ein Ziel wäre, gut ansprechende Patienten zu identifizieren, um eine effiziente, auf diese Patienten abgestimmte Immuntherapie mit möglichst wenig Nebenwirkungen durchführen zu können. Dazu müssen jedoch zuerst die Polymorphismen der Zytokine und deren Rezeptoren genauer analysiert werden [67].

Neben der Verabreichung von Zytokinen müssen auch andere Ansätze wie Inhibition oder Neutralisierung von Zytokinen eruiert werden. Vor allem die TNF- $\alpha$-Blockade scheint ein guter Ansatz zur Hemmung von Entzündungsvorgängen (z.B. auch bei Psoriasis). Die Inhibition kann auf zwei verschiedenen Wegen erreicht werden: durch monoklonale anti-TNF- $\alpha$-Antikörper oder durch lösliche TNF- $\alpha$-Rezeptoren [68-71].

Ein weiterer Fortschritt wird wohl die Kombination von verschiedenen Zytokinen miteinander oder von Zytokinen mit herkömmlichen Chemotherapeutika bringen:

Sequenzielle Chemoimmuntherapie bei metastasierendem Melanom mit Dacarbazin, GM-CSF, IL-2 und INF- $\alpha$ erhöhte in einer Studie von Groenewegen die Zahl aktivierter T-Lymphozyten [72].

Eine Kombinationstherapie mit niedrigdosiertem IL-2 und GMCSF verbesserte den Outcome bei Patienten mit metastasierendem Melanom [73,74]

Kombination von extrakorporaler Photopherese, INF- $\alpha$ und IL-2 könnte bei Sezary-Syndrom effizient sein [75].

Der Einsatz der Zytokine in der Dermatoonkologie ist sehr vielseitig. Das Spektrum reicht von der alleinigen Verabreichung einzelner Zytokine über Kombinationen von verschiedenen Zytokinen oder Kombinationen mit herkömmlichen Chemotherapeutika bis zur Inhibition von Zytokinen. Die bisherigen Resultate reichen leider noch nicht aus, um eine optimale Therapie der verschiedenen Hauttumoren zu definieren. Deshalb sollten diese Substanzen weiterhin bevorzugt in Studien bzw. Therapieoptimierungsprotokollen eingesetzt werden.

\section{Literatur}

${ }^{1}$ Asadullah K, Döcke WD, Reinke P et al. Cytokine determination. Diagnostic significance from the clinical and immunological viewpoint. Dtsch Med Wochenschr 1997; 122: 1424-1431

2 Opal SM, Huber CE. Bench-to-bedside review: Toll-like receptors and their role in septic shock. Crit Care 2002; 6(2): 125-136

${ }^{3}$ Lien E, Ingalls RR. Toll-like receptors. Crit Care Med 2002; 30 (1 Suppl): S1-11

${ }^{4}$ Modlin RL. Activation of toll-like receptors by microbial lipoproteins: role in host defense. J Allergy Clin Immunol 2001; 108 (4 Suppl): S104-S106

${ }^{5}$ Endo TA, Masuhara M, Yokouchi M et al. A new protein containing an SH2 domain that inhibits JAK-kinases. Nature 1997; 387 (6636): 921-924

${ }^{6}$ Heim MH. The Jak-STAT pathway: cytokine signalling from the receptor to the nucleus. J Recept Signal Transduct Res 1999; 19 (1-4): $75-120$

${ }^{7}$ Nashan D, Luger TA. Cytokines: current status and prospects in the treatment of skin tumors. Hautarzt 2001; 52 (8): 691 - 696

8 Stadler R, Mayer-da-Silva A, Bratzke B et al. Interferons in dermatology. J Am Acad Dermatol 1989; 20 (4): 650-656 
9 Lillemeier BF, Koster M, Kerr IM. STAT1 from the cell membrane to the DNA. EMBO J 2001; 20 (10): 2508 - 2517

${ }^{10}$ Jaster R, Tschirch E, Bittorf T et al. Role of STAT5 in interferon-alpha signal transduction in Ba/F3 cells. Cell Signal 1999; 11 (5): 331 - 335

${ }^{11}$ Wu AJ, Chen ZJ, Kann EC et al. Interferon-gamma-induced JAK2 and STAT1 signalling in a human salivary gland cell line. J Cell Physiol 1997; 173 (1): $110-114$

${ }^{12}$ Silvennoinen O, Ihle JN, Schlessinger J et al. Interferon-induced nuclear signalling by Jak protein tyrosine kinases. Nature 1993; 366 (6455): $583-585$

${ }^{13}$ Nashan D, Luger TA. Stand und Perspektiven in der Behandlung von Hauttumoren. Hautarzt 2001; 52 (8): 691 -696

14 Dummer R, Nestle FO, Burg G. Immunotherapy of melanoma. Dtsch Med Wochenschr 2000; 125 (41): 1240-1242

${ }^{15}$ Stadler R, Otte HG, Luger T et al. Prospective randomized multicenter clinical trial on the use of interferon-2a plus acitretin versus interferon-2a plus PUVA in patients with cutaneous T-cell lymphoma stages I and II. Blood 1998; 92 (10): 3578 - 3581

${ }^{16}$ Urosevic M, Dummer R. Immunotherapy for nonmelanoma skin cancer: does it have a future? Cancer 2002; 94 (2): 477-485

${ }^{17}$ Lippmann SM, Parkinson DR, Itri LM et al. 13-cis-retinoid acid and interferon-alpha2a: effective combination therapy for advanced squamous cell carcinoma of the skin. J Nat Cancer Ins 1992; 84: 235-241

${ }^{18}$ Chimenti S, Peris K, Di Cristofaro S et al. Use of recombinant interferon alfa-2b in the treatment of basal cell carcinoma. Dermatology 1995; 190: $214-217$

${ }^{19}$ Ulrich L, Krause M, Brachmann A et al. Successful treatment of angiosarkoma of the scalp by intralesional cytokine therapy and surface irradiation. J Eur Acad Dermatol Venereol 2000; 14 (5): 412 - 415

${ }^{20}$ Kageshita T, Mizuno M, Ono T et al. Growth inhibition of human malignant melanoma transfect with human interferon-beta gene by means of cationic liposomes. Melanoma Res 2001; 11 (4): 337-342

${ }^{21}$ Nagatani T, Okazawa H, Kambara T et al. Effect of natural interferonbeta on the growth of melanoma cell lines. Melanoma Res 1998; 8 (4): 295-299

22 Fierlbeck G, d'Hoedt B, Stroebel W et al. Intralesional therapy of melanoma metastases with recombinant interferon-beta. Hautarzt 1992; 43 (1): $16-21$

${ }^{23}$ Kowalzick L, Mensing H, Weyer U et al. Bowenoid actinic keratosis: therapy with intralesional injection of recombinant beta-interferon. Hautarzt 1992; 43 (6): $373-375$

${ }^{24}$ Mahrle G, Schulze HJ. Recombinant interferon-gamma (rIFN-gamma) in dermatology. J Invest Dermatol 1990; 95 (6 Suppl): 132S - 137S

25 Gross G, Roussaki A, Papendick U. Efficacy of interferons on bowenoid papulosis and other precancerous lesions. J Invest Dermatol 1990; 95 (6 Suppl): 152S-157S

${ }^{26}$ Edwards L, Whiting D, Rogers D et al. The effect of intralesional interferon gamma on basal cell carcinomas. J Am Acad Dermatol 1990; 22 (3): $496-500$

27 Stadler R, Mayer-da-Silva A, Bratzke B et al. Interferons in dermatology. J Am Acad Dermatol 1989; 20 (4): 650-656

${ }^{28}$ Lejeune FJ, Kroon BB, DiFilippo F et al. Isolated limb perfusion: the European experience. Surg Oncol Clin N Am 2001; 10 (4): 821 - 832 ix

${ }^{29}$ Dummer R, Garbe C, Thompson JA et al. A randomized dose escalation study evaluating the safety, tolerability and efficacy of peginterferon alfa-2a (40KD) in patients with metastatic malignant melanoma. ASCO-Abstract

${ }^{30}$ Dummer R, Welters $\mathrm{H}$, Keilholz $\mathrm{U}$ et al. Interleukin 2: immunologic background and clinical use in tumor therapy. Hautarzt 1990; 41 (2): $53-55$

31 Nelson BH, Lord JD, Greenberg PD. Cytoplasmic domains of the interleukin-2 receptor beta and gamma chains mediate the signal for T-cell proliferation. Nature 1994; 369 (6478): 333 - 336

32 Williamson P, Merida I, Gaulton G. Protein and lipid kinase activation cascades in interleukin-2 receptor signalling. Semin Immunol 1993; 5 (5): $337-344$

${ }^{33}$ Liu KD, Greene WC, Goldsmith MA. The alpha chain of the IL-2 receptor determines the species specifity of high-affinity IL-2 binding. Cytokine 1996; 8 (8): 613-621

${ }^{34}$ Minami Y, Kono T, Miyazaki T et al. The IL-2 receptor complex: its structure, function, and target genes. Annu Rev Immunol 1993; 11: $245-268$

${ }^{35}$ Witthuhn BA, Silvennoinen O, Miura O et al. Involvement of the Jak-3 Janus kinase in signalling by interleukins 2 and 4 in lymphoid and myeloid cells. Nature 1994; 370 (6485): $153-157$
${ }^{36}$ Nakamura Y, Russel SM, Mess SA et al. Heterodimerization of the IL-2 receptor beta- and gamma-chain cytoplasmic domains is required for signalling. Nature 1994; 369 (6478): 330-333

${ }^{37}$ Demoulin JB, Renauld JC. Signalling by cytokines interacting with the interleukin-2 receptor gamma chain. Cytokines Cell Mol Ther 1998; 4 (4): $243-256$

38 Tsujino S, Miyazaki T, Kawahara A et al. Critical role of the membraneproximal, proline-rich motif of the interleukin-2 receptor gamma chain in the Jak3-independent signal transduction. Genes cells 1999; 4 (6): $363-373$

${ }^{39}$ Kaplan B, Moy RL. Effect of perilesional injections of PEG-interleukin-2 on basal cell carcinoma. Dermatol Surg 2000; 26 (11): 1037-1040

${ }^{40}$ Rochlitz C, Dreno B, Jantscheff P et al. Immunotherapy of metastatic melanoma by intratumoral injections of Vero cells producing human IL-2: phase II randomized study comparing two dose levels. Cancer Gene Ther 2002; 9 (3): 289-295

${ }^{41}$ Belli F, Arienti F, Sule-Suso J et al. Active immunization of metastatic melanoma patients with interleukin-2-transduced allogeneic melanoma cells: evaluation of efficacy and tolerability. Cancer Immunol Immunother 1997; 45 (2): 119

${ }^{42}$ Spitler LE. Adjuvant therapy of melanoma. Oncology (Huntingt) 2002 16 (1 Suppl 1): $40-48$

${ }^{43} \mathrm{Tu}$ J, Karasavvas N, Heaney ML et al. Molecular characterization of a granulocyte macrophage-colony-stimulating factor receptor alpha subunit-associated protein, GRAP. Blood 2000; 96 (3): 794 - 799

${ }^{44}$ Scott CL, Begley CG. The beta common chain (beta c) of the granulocyte macrophage-colony stimulating facto, interleukin-3 and interleukin-5 receptors. Int J Biochem Cell Biol 1999; 31 (10): 1011 - 1015

${ }^{45}$ Dijkers PF, van Dijk TB, de Groot RP et al. Regulation and function of protein kinase B and MAP kinase activation by the IL-5/GM-CSF/IL-3 receptor. Oncogene 1999; 18 (22): 3334-3342

${ }^{46}$ Nicola NA, Smith A, Robb L et al. The structural basis of the biological actions of the GM-CSF receptor. Ciba Found Symp 1997; 204: 19-27; discussion 27-32

47 Quelle FW, Sato N, Witthuhn BA et al. JAK2 associates with the beta c chain of the receptor for granulocyte-macrophage colony-stimulating factor, and its activation requires the membrane-proximal region. Mol Cell Biol 1994; 14 (7): 4335-4341

${ }^{48}$ Hoeller C, Jansen B, Heere-Ress E et al. Perilesional injection of r-GMCSF in patients with cutaneous melanoma metastases. J Invest Dermatol 2001; 117 (2): 371 - 374

${ }^{49}$ Mahvi DM, Shi FS, Yang NS et al. Immunization by particle-mediated transfer of the granulocyte-macrophage colony-stimulating factor gene into autologous tumor cells in melanoma or sarcoma patients: report of a phase I/IIB study. Hum Gene Ther 2002; 13 (14): $1711-1721$

${ }^{50}$ Dranoff G. GM-CSF-based cancer vaccines. Immunol Rev 2002; 188 (1): $147-154$

${ }^{51}$ Hunger RE, Brand CU, Streit M et al. Successful induction of immune responses against mutant ras in melanoma patients using intradermal injection of peptide and GM-CSF as adjuvant. Exp Dermatol 2001; 10 (3): $161-167$

52 Yue FY, Geertsen R, Hemmi S et al. IL-12 directly up-regulates the expression of HLA class I, HLA class II and ICAM-1 on human melanoma cells: a mechanism for its antitumor activity? Eur J Immunol 1999; 29 (6): $1762-1773$

53 van Rietschoten JG, Smits HH, Westland R et al. Genomic organization of the human interleukin-12 receptor beta2-chain gene. Immunogenetics 2000; 51 (1): 30-36

${ }^{54}$ Nashan D, Luger TA. Cytokines: current status and prospects in the treatment of skin tumors. Hautarzt 2001; 52 (8): 691 - 696

55 Rook AH, Zaki MH, Wysocka M et al. The role for interleukin-12 therapy of cutaneous T cell lymphoma. Ann N Y Acad Sci 2001; 941: $177-184$

56 Rook AH, Wood GS, Yoo EK et al. Interleukin-12 therapy of cutaneous T-cell lymphoma induces lesion regression and cytotoxic T-cell responses. Blood 1999; 94 (3): 902 - 908

57 Yue FY, Cao L, Hemmi S et al. Upregulation of interleukin-12 receptor on peripheral blood mononuclear cells and HLA class I, HLA class II or ICAM-1 on melanoma cells by B7.1 and interleukin-12: a mechanism for immunostimulatory impact of melanoma cells adenoviral transfected with B7.1 and IL12? Melanoma Res 2000; 10 (4): 313-322

${ }^{58}$ Gibson SJ, Lindh JM, Riter TR et al. Plasmacytoid dendritic cells produce cytokines and mature in response to the TLR7 agonists, imiquimod and resiquimod. Cell Immunol 2002; 218 (1-2): $74-86$ 
${ }^{59}$ Stanley MA. Imiquimod and the imidazoquinolones: mechanism of action and therapeutic potential. Clin Exp Dermatol 2002; 27 (7): $571-577$

${ }^{60}$ Hengge UR, Benninghoff B, Ruzicka T et al. Topical immunomodulators - progress towards treating inflammation, infection, and cancer. Lancet Infect Dis 2001; 1 (3): 189-198

61 Tyring S, Conant M, Marini M et al. Imiquimod; an international update on therapeutic uses in dermatology. Int J Dermatol 2002; 41 (11): $810-816$

${ }^{62}$ Stockfleth E, Meyer T, Benninghoff B et al. A randomized, double-blind, vehicle-controlled study to assess $5 \%$ imiquimod cream for the treatment of multiple actinic keratoses. Arch Dermatol 2002; 138 (11): $1498-1502$

${ }^{63}$ Gupta AK, Browne M, Bluhm R. Imiquimod: A Review. J Cutan Med Surg 2002; 6 (6): $554-560$

${ }^{64}$ Bong $A B$, Bonnekoh B, Franke I et al. Imiquimod, a topical immune response modifier, in the treatment of cutaneous metastases of malignant melanoma. Dermatology 2002; 205 (2): 135-138

${ }^{65}$ Ahmed I, Berth-Jones J. Imiquimod: a novel treatment for lentigo maligna. Br J Dermatol 2000; 143 (4): 843-845

${ }^{66}$ Dummer R, Urosevic M, Kempf W et al. Imiquimod induces complete clearance of a PUVA resistant plaque in Mycosis fungoides. Dermatology 2003; 207: $116-118$

${ }^{67}$ Asadullah K, Sterry W, Trefzer U. Cytokine therapy in dermatology. Exp Dermatol 2002; 11 (2): $97-106$

$68 \mathrm{Oh}$ CJ, Das KM, Gottlieb AB. Treatment with antitumor necrosis factor $\alpha$ (TNF- $\alpha$ ) monoclonal antibody dramatically decreases the clinical activity of psoriasis lesions. J Am Acad Dermatol 2000; 42: 829-830
${ }^{69}$ Kirby B, Marsland AM, Carmichael AJ et al. Successful treatment of severe recalcitrant psoriasis with combination infliximab and methotrexate. Clin Exp Dermatol 2001; 26: 27 - 29

${ }^{70}$ Chaudhari U, Romano P, Mulcahy LD et al. Efficacy and safety of infliximab monotherapy for plaque-type psoriasis: a randomised trial. Lancet 2001; 357: 1842 - 1847

${ }^{71}$ Mease PJ, Goffe BS, Metz J et al. Etanercept in the treatment of psoriatic arthritis and psoriasis: a randomised trial. Lancet 2000; 356: $385-390$

${ }^{72}$ Groenewegen G, Bloem A, De Gast GC. Phase I/II study of sequential chemoimmunotherapy (SCIT) for metastatic melanoma: outpatient treatment with dacarbazine, granulocyte-macrophage colony-stimulating factor, low-dose interleukin-2 and interferon-alpha. Cancer Immunol Immunother 2002; 51 (11-12): 630-636

73 O'Day SJ, Boasberg PD, Piro L et al. Maintenance biotherapy for metastatic melanoma with interleukin-2 and granulocyte macrophage-colony stimulating factor improves survival for patients responding to induction concurrent biochemotherapy. Clin Cancer Res 2002; 8 (9): $2775-2781$

${ }^{74}$ Ridolfi L, Ridolfi R, Ascari-Raccagni A et al. Intralesional granulocytemonocyte colony-stimulating factor followed by subcutaneous interleukin-2 in metastatic melanoma: a pilot study in elderly patients. J Eur Acad Dermatol Venereol 2001; 15 (3): 218 - 223

${ }^{75}$ Fritz TM, Kleinhans M, Nestle FO et al. Combination treatment with extracorporeal photopheresis, interferon alfa and interleukin-2 in a patient with the Sezary syndrome. Br J Dermatol 1999; 140 (6): $1144-1147$ 